\title{
Linear-array-based photoacoustic tomography for label-free high- throughput detection and quantification of circulating melanoma tumor cell clusters
}

\author{
Pengfei Hai ${ }^{\text {a, b }}$, Yong Zhou ${ }^{\text {a }}$, Ruiying Zhang ${ }^{\text {a }}$, Jun Ma ${ }^{\text {a }}$, Yang Li ${ }^{\text {a, b }}$, and Lihong V. Wang ${ }^{\text {a, b, * }}$ \\ ${ }^{a}$ Optical Imaging Laboratory, Department of Biomedical Engineering, Washington University in St. \\ Louis, One Brookings Drive, St. Louis, Missouri 63130 \\ ${ }^{\mathrm{b}}$ Caltech Optical Imaging Laboratory, Department of Medical Engineering, California Institute of \\ Technology, 1200 E California Blvd, Pasadena, CA 91125 \\ *Corresponding author:1vw@caltech.edu
}

\begin{abstract}
Circulating tumor cell (CTC) clusters arise from multicellular grouping in the primary tumor and elevate the metastatic potential by 23 to 50 fold compared to single CTCs. High throughout detection and quantification of CTC clusters is critical for understanding the tumor metastasis process and improving cancer therapy. In this work, we report a lineararray-based photoacoustic tomography (LA-PAT) system capable of label-free high-throughput CTC cluster detection and quantification in vivo. LA-PAT detects CTC clusters and quantifies the number of cells in them based on the contrast-to-noise ratios (CNRs) of photoacoustic signals. The feasibility of LA-PAT was first demonstrated by imaging CTC clusters ex vivo. LA-PAT detected CTC clusters in the blood-filled microtubes and computed the number of cells in the clusters. The size distribution of the CTC clusters measured by LA-PAT agreed well with that obtained by optical microscopy. We demonstrated the ability of LA-PAT to detect and quantify CTC clusters in vivo by imaging injected CTC clusters in rat tail veins. LA-PAT detected CTC clusters immediately after injection as well as when they were circulating in the rat bloodstreams. Similarly, the numbers of cells in the clusters were computed based on the CNRs of the photoacoustic signals. The data showed that larger CTC clusters disappear faster than the smaller ones. The results prove the potential of LA-PAT as a promising tool for both preclinical tumor metastasis studies and clinical cancer therapy evaluation.
\end{abstract}

Keywords: photoacoustic tomography, cancer metastasis, circulating tumor cell, cancer therapy

\section{INTRODUCTION}

The majority of cancer deaths are associated with metastasis, the process in which the cancer cells spread from a primary site to distant organs and lead to the growth of new tumors there ${ }^{1}$. During metastasis, cancer cells need to survive the intravasation, circulation in the blood, extravasation, and secondary growth in the distant organs. Thus, the rare circulating cancer cells (CTCs) are the key determinants of the metastatic propensity ${ }^{2}$. While previously CTCs are considered to exist mostly in single cells, recent studies have found that CTC clusters also exist and elevate the metastatic potential by 23 to $50 \mathrm{fold}^{3}$. They arise from the multicellular grouping in the primary tumor and can traverse capillary-sized vessels ${ }^{4}$. Detection and quantification of CTC clusters will provide valuable insights into metastasis as well as cancer therapy.

Taking advantage of tumor-specific physical and biological properties, such as sizes and surface biomarkers, multiple technologies have been developed to capture and isolate single CTCs and CTC clusters ${ }^{5-7}$. However, most of these existing technologies require blood to be drawn to isolate and capture CTCs ex vivo, reducing the effective CTC detection sensitivity. Optical imaging technologies, such as confocal microscopy, in vivo flow cytometry, and optical coherence tomography, have been applied to detect CTCs in vivo ${ }^{8-11}$. However, suffering from strong optical scattering in biological tissue, these techniques have shallow penetration, limiting them to imaging only small blood vessels, which results in low throughput for CTC detection.

Photons Plus Ultrasound: Imaging and Sensing 2017, edited by Alexander A. Oraevsky, Lihong V. Wang, Proc. of SPIE Vol. 10064, 100641P · @ 2017 SPIE · CCC code: 1605-7422/17/\$18 · doi: 10.1117/12.2252819 
By integrating optical excitation with acoustic detection, photoacoustic tomography (PAT) combines rich optical absorption contrasts with high ultrasonic spatial resolution at depths ${ }^{12}$. With $100 \%$ relative sensitivity to optical absorption, i.e., a given percentage change in the optical absorption yields the same percentage change in the photoacoustic signal, PAT achieves structural, functional, metabolic, and mechanical imaging of biological tissue ${ }^{13-17}$. Taking advantage of the strong optical absorption of melanin, photoacoustic techniques have been successfully used for imaging and sensing melanoma CTCs. Photoacoustic flowmetry successfully detected circulating melanoma cells in human blood ${ }^{18,19}$. Photoacoustic flow cytometry enables long-term monitoring of melanoma CTCs in vivo. However, these sensing techniques provide inadequate spatial resolution to further characterize melanoma $\mathrm{CTCs}^{20}$. The microscopic implementation of PAT, optical-resolution photoacoustic microscopy (OR-PAM), images single melanoma CTCs in the blood flows with high spatial-temporal resolution ${ }^{21}$. While providing valuable insights into CTCs on a single-cell level, OR-PAM suffers from low throughput.

Here, we apply a linear-array-based photoacoustic tomography (LA-PAT) system for label-free high-throughput melanoma CTC cluster detection and quantification in vivo. Exploiting the strong optical absorption of melanin in the melanoma tumor cells, LA-PAT can achieve label-free detection of melanoma CTC clusters in vivo. By analyzing the contrast-to-noise ratios (CNRs) of the photoacoustic signals, LA-PAT can quantify the number of cells in the CTC clusters and study their circulating kinetics in the bloodstream.

\section{MATERIALS AND METHODS}

\subsection{Experimental system}

To image melanoma CTC clusters, we applied an LA-PAT system based on a handheld probe and improved the image reconstruction ${ }^{22,23}$. A tunable optical parametric oscillator laser $(680$ to $970 \mathrm{~nm}, 20-\mathrm{Hz}$ pulse repetition rate) was used for illumination. The laser beam was coupled into a fiber optical bundle that bifurcated into two optical fiber bundles. Laser beams coming out of the two optical fiber bundle strips $\left(20 \times 1.25 \mathrm{~mm}^{2}\right)$ excited the object at an angle of incidence of 30 degree relative to the imaging plane [Figs. 1(a) and 1(b)]. During the experiments, the fluence on the tissue surface was about $10 \mathrm{~mJ} / \mathrm{cm}^{2}$, well within the $20 \mathrm{~mJ} / \mathrm{cm}^{2}$ safety limit set by the American National Standards Institute. The generated photoacoustic waves were detected by a linear array ultrasonic transducer (Visualsonics, Inc., LZ250, 21-MHz center frequency, $78 \%$ one-way bandwidth, 256 elements, $23 \times 3 \mathrm{~mm}^{2}$ array size). The spatial resolutions of the system were $119 \mu \mathrm{m}$ in the lateral direction, $86 \mu \mathrm{m}$ in the axial direction, and $1237 \mu \mathrm{m}$ in the elevational direction ${ }^{24}$. Because there were only 64 channels in the data acquisition unit, four-to-one multiplexing was applied during image acquisition. For each laser pulse, the generated photoacoustic signals were captured sequentially by a quarter segment of the linear array (i.e., elements 1 to 64,65 to 128,129 to 192 , and 193 to 256). Once the data were acquired from all four quarter segments, a two-dimensional photoacoustic image was reconstructed with the universal back-projection algorithm developed by our group ${ }^{25}$. The reconstructed photoacoustic images were displayed in an imaging station (Vevo LAZR, Visualsonics, Inc.). Determined by the $20-\mathrm{Hz}$ laser repetition rate and the four-to-one multiplexing in image acquisition, the frame rate was 5 frames/s.

(a)

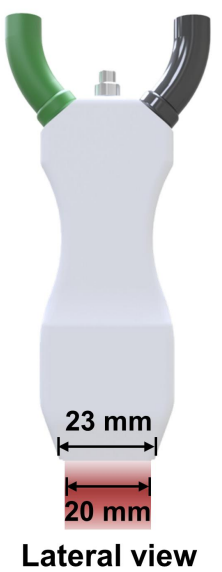

(b)

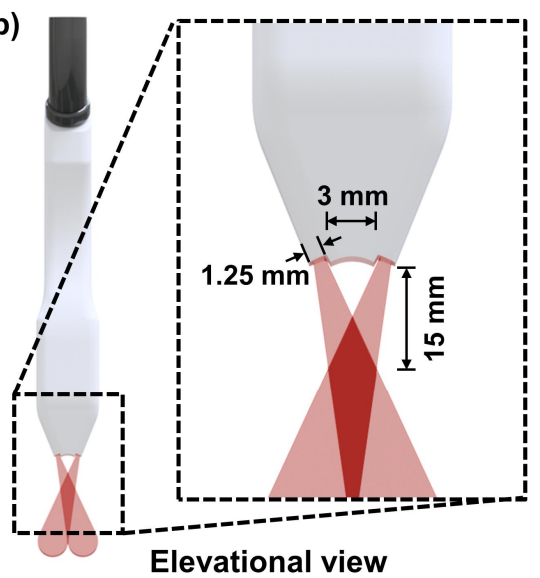

Figure 1. Front and side views of the linear-array-based photoacoustic tomography (LA-PAT) system with a handheld probe. 


\subsection{Tumor cell culture and cluster preparation}

B16F10 melanoma tumor cells obtained from $\mathrm{ATCC}^{\circledR}$ were cultured in Dulbecco's modified Eagle's medium (Invitrogen) supplemented with $10 \%$ fetal bovine serum (Invitrogen) at $37^{\circ} \mathrm{C}$ in air with $5 \% \mathrm{CO} 2$. Upon reaching $80 \%$ confluence, cells growing in a monolayer were incubated with $0.25 \%$ trypsin-EDTA solution (Invitrogen) to generate floating cell clusters of various sizes. After adding serum-containing medium to neutralize trypsin, the floating cell clusters were handled in two ways. To mimic melanoma CTC clusters of various sizes, floating cell clusters totaling $1 \times 10^{6}$ cells were directly suspended in $1 \mathrm{~mL}$ of bovine blood for ex vivo experiments or in $1 \mathrm{~mL}$ of collected rat blood for tail vein injection. To generate melanoma cell clusters of a certain size for CNR analysis, instead of directly suspending the floating cell clusters in blood, the clusters were mechanically dissociated by pipetting to generate a single-cell suspension. The single cells were then suspended in a blood-agar mixture at a desired concentration and then cooled to become solid. The concentration was controlled so that within each resolution voxel of LA-PAT there was a certain number of melanoma cells, i.e., 5, 10, 20, 30, 40, or 50. The solid mixture was then mechanically dissociated into particles smaller than the resolution voxel of LA-PAT to generate cell clusters of a certain size. The particles were then suspended in $1 \mathrm{~mL}$ of bovine blood for CNR analysis.

\subsection{Phantom preparation}

Silicone microtubes with $0.76 \mathrm{~mm}$ inner diameter (11-189-15C, Fisher Scientific) were perfused with bovine blood (905, Quad-Five) to mimic blood vessels. The microtubes were embedded $3 \mathrm{~mm}$ deep in tissue-mimicking gelatin phantoms. Optical scattering similar to that in biological tissue was achieved by adding $1 \%$ intralipid to the gelatin phantoms. Melanoma tumor cell clusters suspended in bovine blood were pumped through the microtubes with a syringe. A syringe pump (BSP-99M, Braintree Scientific) controlled the blood flow speed.

\subsection{Experimental animals and CTC cluster injection}

All experimental animal procedures were carried out in conformity with the laboratory animal protocol approved by the Animal Studies Committee at Washington University in St. Louis. Male nude rats (Hsd:RH-Foxn $1^{\text {rnu }} / F_{\text {oxn }}{ }^{+}$, Harlan Co.; 12- to 13-weeks old; 330 to $350 \mathrm{~g}$ body weight) were used for all in vivo experiments. Throughout the experiments, the rats were kept under anesthesia with $2 \%$ vaporized isoflurane. A homemade animal holder immobilized the rats during the experiments.

\section{RESULTS}

\subsection{Optimal wavelength for CTC cluster detection}

To maximize the melanoma CTC cluster detection sensitivity, the illumination wavelength should be chosen carefully. Since the major optical absorber in blood is hemoglobin, we compared the optical absorption spectra of melanin, major optical absorber in melanoma tumor cells, and hemoglobin [Fig. 2(a)]. The optical absorption coefficient ratio of melanin and venous blood with oxygen saturation level of $85 \%$ was calculated [Fig. 2(b)]. The venous blood was chosen because veins are ideal targets for detecting melanoma tumor cells with shallow depth in tissue and large blood volume. Based on the results, $680 \mathrm{~nm}$ was chosen to maximize the CTC cluster detection sensitivity.

\subsection{CNR analysis of melanoma tumor cell clusters}

To detect and quantify CTC clusters, we first used LA-PAT to image melanoma tumor cell clusters of known sizes and analyzed their CNRs. Clusters with an average of 5, 10, 20, 30, 40, and 50 cells were suspended in blood and imaged by LA-PAT. The CNR for each cluster was calculated [Fig. 3(a)]. The average CNRs were plotted as a function of the number of cells in the clusters, as measured with an optical microscope (OM) (Eclipse TS100, Nikon) [Fig. 3(b)]. The experimental data were fitted to a linear function

$$
C N R=0.46 \times N
$$

Here, $N$ represents the number of cells in a melanoma tumor cell cluster. Based on a 6-dB CNR threshold, a minimum of four cells can be resolved although this minimum was not directly observed. To verify the relationship between the CNR and the number of cells in the clusters, another independent experiment was performed. Melanoma tumor cell clusters consisting of a uniform but unknown number of cells were equally divided into six groups. Three randomly chosen groups were suspended in blood and imaged by LA-PAT. The numbers of cells in the clusters were calculated based on the CNRs and the relationship above. The other three groups were examined with an OM to determine the numbers of 
cells in the clusters. The number of cells in the melanoma tumor cell clusters measured by LA-PAT and optical microscopy agreed well with each other [Fig. 4], confirming the relationship between the numbers of cells in the clusters and the CNRs in LA-PAT.

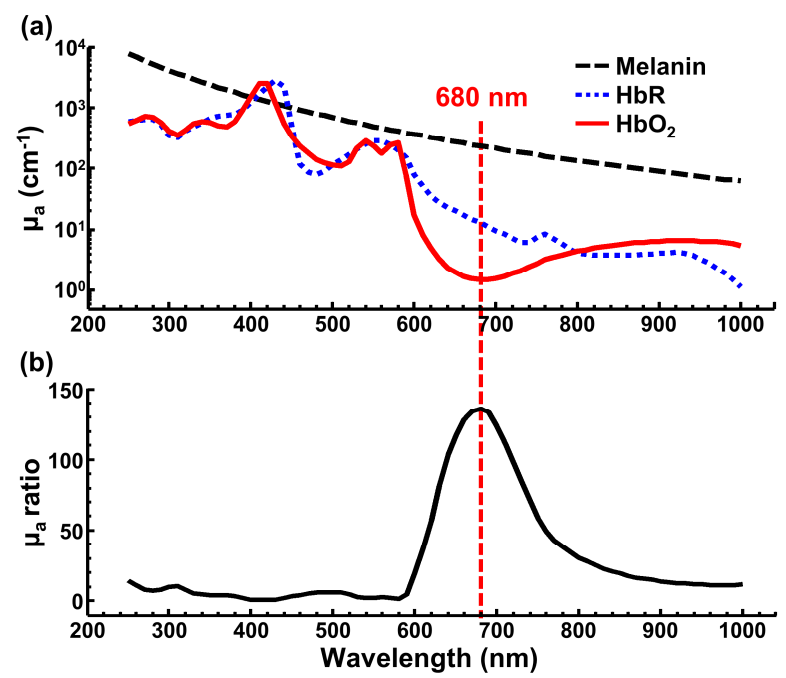

Figure 2. Absorption spectra of melanin and hemoglobin and absorption coefficient ratio of melanin and blood with oxygen saturation $\left(\mathrm{sO}_{2}\right)$ level of $85 \%$. (a) Absorption spectra of melanin and hemoglobin from $250 \mathrm{~nm}$ to $1000 \mathrm{~nm}$. (b) Absorption coefficient ratio of melanin and blood with $\mathrm{sO}_{2}$ of $85 \%$. An excitation wavelength of $680 \mathrm{~nm}$ was chosen to maximize the melanoma CTC detection sensitivity.

(a)

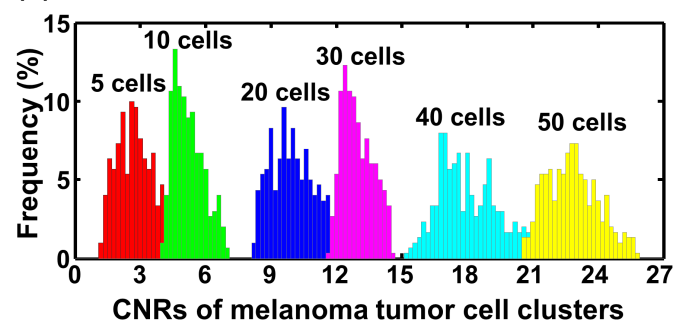

(b)

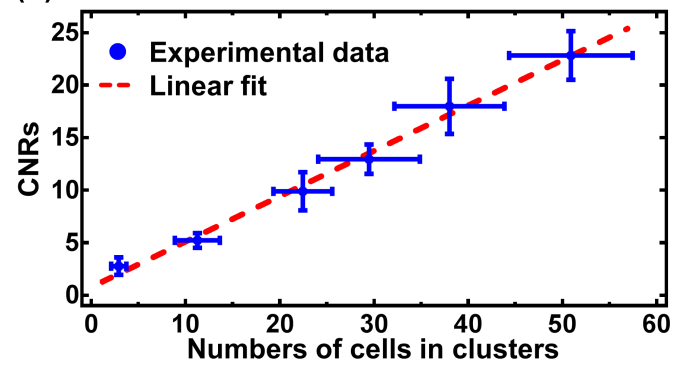

Figure 3. Contrast-to-noise ratio (CNR) analysis of melanoma tumor cell clusters. (a) CNR distribution of melanoma tumor cell clusters of different sizes by LA-PAT. (b) Average CNRs as a function of the numbers of cells in melanoma tumor cell clusters.

\subsection{LA-PAT of CTC clusters ex vivo}

We then demonstrated the ability of LA-PAT to detect and quantify CTC clusters ex vivo. A mixture of melanoma tumor cell clusters of various sizes was equally divided into six groups. Three randomly chosen groups were suspended in blood, pumped through microtubes to mimic flowing melanoma CTC clusters, and imaged by LA-PAT. Each melanoma tumor cell cluster was captured by LA-PAT multiple times, and the average CNR was used to calculate the number of cells in the cluster. Based on the calculated number of cells in the clusters, the melanoma tumor cell clusters were 
divided into three categories: $\leqslant 10,11$ to 20 , and $\geqslant 21$. Typical photoacoustic images of the three categories are shown in Figs. 5(a)-5(c). The other three groups were measured with an OM to determine the numbers of cells in the clusters and were divided into the same three categories. The size distributions of the clusters measured by LA-PAT and optical microscopy agreed well with each other with a correlation coefficient of 0.96 [Fig. 5(d)], and the results further demonstrated the ability of LA-PAT to detect and quantify melanoma CTC clusters.

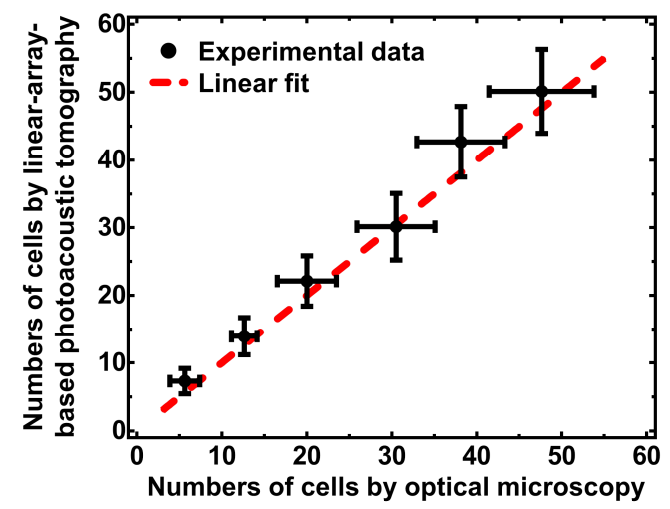

Figure 4. The numbers of cells in melanoma tumor cell clusters measured by LA-PAT agreed well with those measured by optical microscopy (OM).
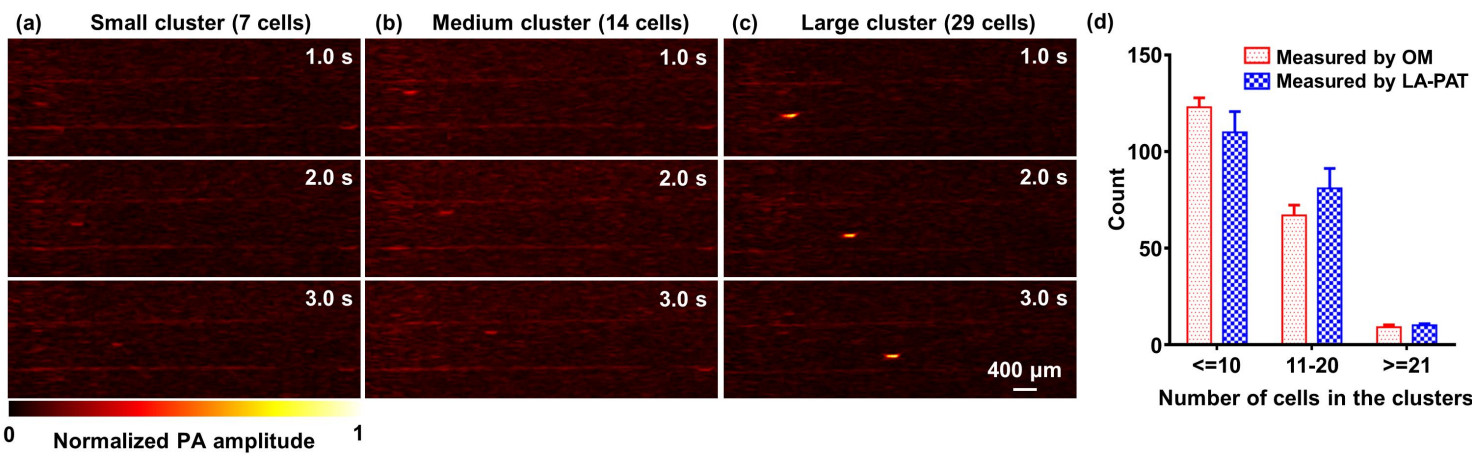

Figure 5. LA-PAT of CTC clusters ex vivo. LA-PAT of flowing melanoma tumor cell clusters with (a) 7 cells, (b) 14 cells, and (c) 29 cells. The cell numbers are calculated based on Eq. (1). (d) Distributions of the numbers of cells in melanoma tumor cell clusters, as measured by LA-PAT and OM.

\subsection{LA-PAT of CTC clusters in vivo}

To demonstrate the feasibility of LA-PAT in vivo, we imaged melanoma CTC clusters injected into rats. Melanoma tumor cell clusters suspended in $1 \mathrm{~mL}$ of rat blood at a concentration of $1 \times 10^{6}$ cells $/ \mathrm{mL}$ were injected into the same rat from which the blood was collected a few minutes earlier. The rat tail vein was then monitored by LA-PAT for 45 min to study the circulation kinetics and the clearance rates of CTC clusters. CTC clusters were detected by LA-PAT immediately after injection and during circulating in the rat bloodstreams [Figs. 6(a) and 6(b)]. The numbers of cells in the CTC clusters were calculated based on the CNRs. The CTC clusters were categorized into three groups based on the calculated number of cells and the clearance curves of the three groups are shown in Fig. 6(c). Larger CTC clusters disappeared faster than smaller ones.

\section{DISCUSSIONS AND CONCLUSIONS}

Compared with single CTCs, CTC clusters are rarer, but have a higher metastatic potential. CTC clusters have a faster clearance rate, i.e., a shorter lifetime, in the bloodstream than single CTCs, making them even more difficult to detect. Here, we applied LA-PAT to detect and quantify melanoma CTC clusters in rat in vivo. Since the spatial resolutions of LA-PAT were not enough to resolve single melanoma tumor cells, the numbers of cells in the CTC clusters were quantified based on the CNRs. A linear relationship between the CNRs and the numbers of cells in the CTC clusters was 
found and verified. In ex vivo experiments, LA-PAT quantified the numbers of cells in the CTC clusters, and the results were validated by an OM. In experiments in vivo, LA-PAT detected and quantified melanoma CTC clusters immediately after injection, as well as when they were circulating in the rat bloodstreams. The results by LA-PAT also showed that larger CTC clusters disappeared faster than smaller ones.
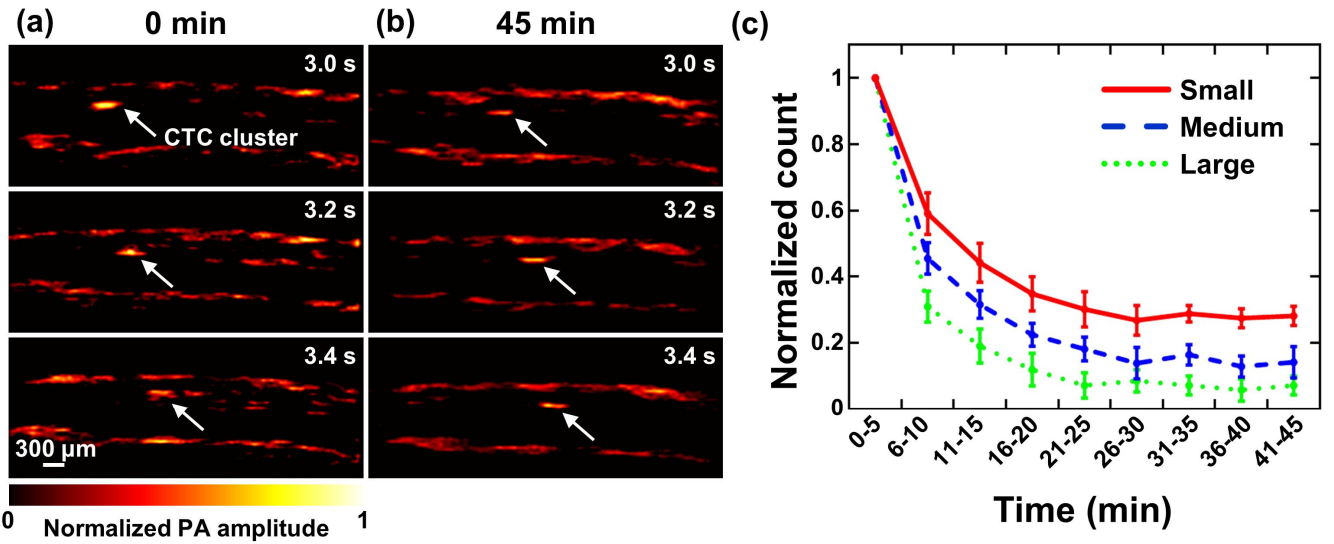

Figure 6. LA-PAT of CTC clusters in rat tail veins in vivo. (a) LA-PAT of CTC clusters in a rat tail vein in vivo immediately after injection. (b) LA-PAT of CTC clusters in the rat tail vein in vivo $45 \mathrm{~min}$ after injection. (c) Clearance curves of small ( $\leqslant 10$ cells), medium ( 11 to 20 cells), and large ( $\geqslant 21$ cells) CTC clusters in rat bloodstreams.

The melanoma CTC detection sensitivity of LA-PAT is determined by the CNR. Because the noise is dominated by the fluctuating counts of red blood cells in each resolution voxel, the detection sensitivity is largely dependent on the spatial resolutions of LA-PAT. On one hand, to achieve single cell sensitivity in CTC detection, a higher frequency ultrasonic transducer could be used at the expense of penetration depth. On the other hand, to achieve deeper imaging, a lower frequency ultrasonic transducer can be employed, at the expense of CTC detection sensitivity. The CTC detection sensitivity can be further improved by performing spectral unmixing using multi-wavelength illumination. To achieve multi-wavelength imaging of flowing CTCs, a second laser with a different wavelength needs to be incorporated into the system.

It is worth pointing out that currently LA-PAT detects only CTCs originating from primary tumors in which cells express melanin. To detect CTCs originating from amelanotic melanoma tumors, other tumor-specific physiological properties, such as sizes and surface biomarkers, can be utilized. The quantification of CTC cluster sizes is indeed based on the assumption that the melanin content is relatively uniform in the tumor cells. This assumption often holds true for tumor cells originating from the same primary tumor. However, if there are several primary tumors, it may affect the accuracy of quantifying CTC cluster sizes.

LA-PAT has the potential to be a powerful tool for both preclinical tumor metastasis study and clinical cancer diagnosis and therapy. As a useful technique for researchers and scientists to better understand the relationship between CTCs and tumor metastasis, LA-PAT can employ ultrasonic transducers of different frequencies for target vessels at various depths and different animal models. Other physiological parameters, including vessel diameter and blood flow speed, can be measured concurrently for further understanding of CTC flow dynamics. LA-PAT can also be used by clinicians to monitor the changes of CTC concentrations in patients' circulatory systems and to evaluate the outcome of cancer therapy. To further improve LA-PAT for such clinical applications, an automatic CTC detection and counting algorithm should be developed.

Metastasis is a complex biological process that involves multiple steps and many parameters. Photoacoustic imaging, with the ability to quantify numerous parameters at multiple scales based on the same optical absorption contrast, can provide comprehensive information about tumor metastasis. OR-PAM can study CTCs on a single-cell level ${ }^{21}$. LA-PAT has been demonstrated to detect and quantify CTC clusters. In addition, photoacoustic imaging can measure other important biological parameters, including tumor depth, the oxygen saturation of hemoglobin, metabolic rate, and tumor stiffness $^{26-29}$, all of which are closely related to metastasis ${ }^{30-32}$. With the capability to detect and quantify CTC clusters and the potential to provide multidimensional information on tumor metastasis, LA-PAT is a promising tool for both preclinical cancer metastasis study and clinical tumor therapy. 


\section{ACKNOWLEDGMENTS}

We thank Eric Zhang for his help in cell cluster preparation. We also thank Professor James Ballard for a close reading of this paper. This work was sponsored by the US National Institutes of Health grant Nos. DP1 EB016986 (NIH Director's Pioneer Award), R01 CA186567 (NIH Director's Transformative Research Award), and S10 RR026922, and US National Science Foundation IDBR award 1255930. L. V. Wang has a financial interest in Microphotoacoustics, Inc., which, however, did not support this work.

\section{REFERENCES}

[1] Nguyen, D. X., Bos, P. D., and Massagué, J., "Metastasis: from dissemination to organ-specific colonization," Nat. Rev. Cancer 9(4), 274-284 (2009).

[2] Fidler, I. J., "The pathogenesis of cancer metastasis: the 'seed and soil' hypothesis revisited," Nat. Rev. Cancer 3(6), 453-458 (2003).

[3] Aceto, N. et al., "Circulating tumor cell clusters are oligoclonal precursors of breast cancer metastasis," Cell 158(5), 1110-1122 (2014).

[4] Au, S. H. et al., "Clusters of circulating tumor cells traverse capillary-sized vessels," Proc. Natl. Acad. Sci. 113(18), 4947-4952 (2016).

[5] Yu, M., Stott, S., Toner, M., Maheswaran, S. and Haber, D. A., "Circulating tumor cells: approaches to isolation and characterization," J. Cell Biol. 192(3), 373-382 (2011).

[6] Nagrath, S. et al., "Isolation of rare circulating tumour cells in cancer patients by microchip technology," Nature 450(7173), 1235-1239 (2007).

[7] Sarioglu A. F. et al., "A microfluidic device for label-free, physical capture of circulating tumor cell clusters," Nat. Methods 12(7), 685-691 (2015).

[8] Sahai E., "Illuminating the metastatic process," Nat. Rev. Cancer 7(10), 737-749 (2007).

[9] Seo, H., Hwang, Y., Choe, K. and Kim, P., "In vivo quantitation of injected circulating tumor cells from great saphenous vein based on video-rate confocal microscopy," Biomed. Opt. Express 6(6), 2158-2167 (2015).

[10] Georgakoudi, I., Solban, N., Novak, J., Rice, W. L., Wei, X., Hasan, T. and Lin, C. P., "In vivo flow cytometry: a new method for enumerating circulating cancer cells," Cancer Res. 64(15), 5044-5047 (2004).

[11] Azarin S. M. et al., "In vivo capture and label-free detection of early metastatic cells," Nat. Commun. 6, 8094 (2015).

[12] Wang, L. V. and Hu, S., "Photoacoustic tomography: in vivo imaging from organelles to organs," Science 335(6075), 1458-1462 (2012).

[13] Hai, P., Yao, J., Maslov, K.I., Zhou, Y. and Wang, L.V., "Near-infrared optical-resolution photoacoustic microscopy,” Opt. Lett. 39(17), 5192-5195 (2014).

[14] Ma, J., Shi, J., Hai, P., Zhou, Y., and Wang, L. V., "Grueneisen relaxation photoacoustic microscopy in vivo," J. Biomed. Opt. 21(6), 066005 (2016).

[15] Wang, L. V., and Yao, J., "A practical guide to photoacoustic tomography in the life sciences," Nat. Methods. 13(8), 627-638 (2016).

[16] Wong, T. T. W., Zhang, R., Hai, P., Zhang, C., Pleitez, M. A., Aft, R., Novak, D., and Wang, L. V., "Fast label-free multi-layered histology-like imaging of human breast cancer by photoacoustic microscopy," Science Advances (under review).

[17] Hai, P., Yao, J., Li, G., Li, C., and Wang, L. V., “Photoacoustic elastography,” Opt. Lett. 41(4), 725-728 (2016).

[18] Weight, R. M., Viator, J. A., Dale, P. S., Caldwell, C. W. and Lisle, A. E., "Photoacoustic detection of metastatic melanoma cells in the human circulatory system," Opt. Lett. 31(20), 2998-3000 (2006).

[19] Weight, R.M., Dale, P.S. and Viator, J.A., "Detection of circulating melanoma cells in human blood using photoacoustic flowmetry," in Annual Int. Conf. of the IEEE Engineering in Medicine and Biology Society (EMBC), 106-109 (2009).

[20] Galanzha, E.I. and Zharov, V.P., “Photoacoustic flow cytometry,” Methods 57(3), 280-296 (2012).

[21] Wang, L., Yao, J., Zhang, R., Xu, S., Li, G., Zou, J. and Wang, L.V., "Photoacoustic imaging of single circulating melanoma cells in vivo," Proc. SPIE 9323, 93230A (2015). 
[22] Needles, A., Heinmiller, A., Sun, J., Theodoropoulos, C., Bates, D., Hirson, D., Yin, M., and Foster, F.S., "Development and initial application of a fully integrated photoacoustic micro-ultrasound system," IEEE Trans. Ultrason. Ferroelectr. Freq. Control 60(5), 888-897 (2013).

[23] Hai, P., Zhou, Y., Zhang, R., Ma, J., Li, Y., Shao, J. Y., and Wang, L. V., "Label-free high-throughput detection and quantification of circulating melanoma tumor cell clusters by linear-array-based photoacoustic tomography," J. Biomed. Opt. 22(4), 041004 (2016).

[24] Hai, P., Zhou, Y., Liang, J., Li, C. and Wang, L.V., "Photoacoustic tomography of vascular compliance in humans," J. Biomed. Opt. 20(12), 126008 (2015).

[25] Xu, M., and Wang, L. V., "Universal back-projection algorithm for photoacoustic computed tomography," Phys. Rev. E. 71(1), 016706 (2005).

[26] Zhou, Y., Tripathi, S. V., Rosman, I., Ma, J., Hai, P., Linette, G. P., Council, M. L., Fields, R. C., Wang, L. V., and Cornelius, L. A., "Noninvasive determination of melanoma depth using a handheld photoacoustic probe," J. Invest. Dermatol. (in press).

[27] Zhang, H. F., Maslov, K., Stoica, G. and Wang, L. V., "Functional photoacoustic microscopy for high-resolution and noninvasive in vivo imaging," Nat. Biotechnol. 24 (7), 848-851 (2006).

[28] Yao, J., Xia, J., Maslov, K. I., Nasiriavanaki, M., Tsytsarev, V., Demchenko, A. V. and Wang, L.V., "Noninvasive photoacoustic computed tomography of mouse brain metabolism in vivo," NeuroImage 64(1), 257-266 (2013).

[29] Hai, P., Zhou, Y., Gong, L., and Wang, L. V., "Quantitative photoacoustic elastography in humans," J. Biomed. Opt. 21(6), 066011 (2016).

[30] Luke, G. P. and Emelianov, S. Y., "Label-free detection of lymph node metastases with US-guided functional photoacoustic imaging," Radiology 277(2), 435-442 (2015).

[31] Fong Y. et al., "Breast-cancer-secreted miR-122 reprograms glucose metabolism in premetastatic niche to promote metastasis," Nat. Cell Biol. 17(2), 183-194 (2015).

[32] Fenner, J., Stacer, A. C., Winterroth, F., Johnson, T. D., Luker, K. E. and Luker, G. D., "Macroscopic stiffness of breast tumors predicts metastasis," Sci. Rep. 4, 5512 (2014). 